\title{
Odd Perfect Numbers Not Divisible By 3. II
}

\section{By Masao Kishore*}

\begin{abstract}
We prove that odd perfect numbers not divisible by 3 have at least eleven distinct prime factors.
\end{abstract}

1. $N$ is called a perfect number if $\sigma(N)=2 N$, where $\sigma(N)$ is the sum of positive divisors of $N$. Twenty-seven even perfect numbers are known; however, no odd perfect (OP) numbers have been found.

Suppose $N$ is OP and $\omega(N)$ is the number of distinct prime factors of $N$. Gradstein (1925), Kühnel (1949), Weber (1951), and the author (1978, [5]) proved that $\omega(N) \geqslant 6$. Pomerance (1972, [7]) and Robbins (1972) proved that $\omega(N) \geqslant 7$. Hagis $(1975,[2])$ and Chein $(1978,[1])$ proved that $\omega(N) \geqslant 8$.

Hagis and McDaniel [3] proved that the largest prime factor of $N \geqslant 100129$, and Pomerance [8] proved that the second largest prime factor of $N \geqslant 139$.

If $3 \nmid N$, then Kanold (1949) proved that $\omega(N) \geqslant 9$, and the author (1977, [4]) proved that $\omega(N) \geqslant 10$.

In this paper we prove

THEOREM. If $N$ is $O P$ and $3 \nmid N$, then $\omega(N) \geqslant 11$.

2. In the remainder of this paper we assume that $N$ is OP and

$$
N=\prod_{i=1}^{10} p_{i}^{a_{i}},
$$

where $p_{i}$ 's are primes, $5 \leqslant p_{1}<\cdots<p_{10}$ and $a_{i}$ 's are positive integers, and we will get a contradiction. We write $p_{i}^{a_{i}} \| N$ and $a_{i}=V_{p_{i}}(N)$.

The following lemmas were proved in [4] and [7]:

Lemma 1. Suppose $p^{a} \| N$. Then

(a) All a's are even except for one $a$ in which case $a \equiv p \equiv 1(4)$. We write $\pi$ for $p$.

(b) If $p \equiv 1$ (3), $a \neq 2$ (3).

(c) If $p \equiv 1$ (4) and $p \equiv 2$ (3), then $p \neq \pi$.

LEMMA 2. Suppose $q=5$ or 17 and $p^{a} \| N$. Then

$$
V_{q}\left(\sigma\left(p^{a}\right)\right)= \begin{cases}V_{q}(a+1) & \text { if } p \equiv 1(q), \\ V_{q}(p+1)+V_{q}(a+1) & \text { if } p \equiv-1(q) \text { and } p=\pi, \\ 0 & \text { otherwise. }\end{cases}
$$

Received November 2, 1981; revised June 21, 1982.

1980 Mathematics Subject Classification. Primary 10A20.

${ }^{*}$ Current address: Department of Mathematics, East Carolina University, Greenville, North Carolina 27834 . 
Lemma 3. Suppose $p^{a} \| N, q$ is a prime and $q^{b} \| a+1$. Then $N$ is divisible by at least $c$ distinct primes $\equiv 1(q)$ other than $p$, where $c=b$ if $q^{b}=a+1$, and $c=2 b$ if $q^{b} \neq a+1$.

The proof of the next lemma is similar to that of Lemma 6 in [4].

LeMmA 4. $p_{1}=5, p_{2}=7, p_{3}=11, p_{4} \leqslant 17, p_{5} \leqslant 23, p_{6} \leqslant 37, p_{7} \leqslant 107, p_{9} \geqslant 139$, $p_{10} \geqslant 100129$. If $p_{7} \geqslant 103$, then $p_{8} \leqslant 113$.

Lemma 5. Suppose $p, q$ are odd primes, $a, b$ are positive integers, $p^{b} \mid q+1, p \geqslant 5$ and $2 b \geqslant a$. Then $q \nmid \sigma\left(p^{a}\right)$.

Proof. Since $p$ and $q$ are odd primes, $q \geqslant 2 p^{b}-1$. Suppose $\sigma\left(p^{a}\right)=m q$ for some integer $m$. Then $a \geqslant b$ and

$$
\sigma\left(p^{b-1}\right)+m \equiv \sigma\left(p^{a}\right)+m=m(q+1) \equiv o\left(p^{b}\right) .
$$

Hence

$$
m \geqslant p^{b}-\sigma\left(p^{b-1}\right)=\left(p^{b+1}-2 p^{b}+1\right) /(p-1),
$$

and

$$
\begin{aligned}
\sigma\left(p^{a}\right) & =m q \geqslant\left(2 p^{b}-1\right)\left(p^{b+1}-2 p^{b}+1\right) /(p-1) \\
& =\left(2 p^{2 b+1}-4 p^{2 b}-p^{b+1}+4 p^{b}-1\right) /(p-1) \\
& >\left(p^{2 b+1}-1\right) /(p-1)=\sigma\left(p^{2 b}\right) \geqslant \sigma\left(p^{a}\right),
\end{aligned}
$$

because $p \geqslant 5$ and $2 b \geqslant a$, a contradiction. Q.E.D.

Remark. Lemma 5 also holds if $p=3$ and $2 b>a$.

The next lemma is due to Hagis.

LEMMA 6. Suppose $p=5$ or 17 and $p^{a}$ is a component of an OP number. Then $\sigma\left(p^{a}\right)$ has at least one prime factor $\geqslant 100129$ except

(a) if $p=5, a=1,2,4,5,6,8,9,13,14,17,26,29$.

(b) If $p=17, a=1,2,4,5,9$.

CoRollaRY 6. Suppose $p=5$ or 17 and $p^{a} \| N$. Then $\sigma\left(p^{a}\right)$ has at least one prime factor $\geqslant 100129$ except

(a) if $p=5, a=2,4,6,8$,

(b) if $p=17, a=2,4$.

Proof. We can easily show that $5^{14} \nVdash N$ and $5^{26} \nVdash N$ because $\sigma\left(5^{14}\right)=$ $11 \cdot 13 \cdot 71 \cdot 181 \cdot 1741$ and $\sigma\left(5^{26}\right)=19 \cdot 31 \cdot 109 \cdot 271 \cdot 829 \cdot 4159 \cdot 31051$. Then Corollary 6 follows from Lemmas 1 and 6. Q.E.D.

LEMMA 7. If $17^{a} \| N$ and $a \geqslant 8$, then $p_{9} \geqslant 100129, p_{10} \geqslant 2 \cdot 17^{a-3}-1>2 \cdot 10^{6}$, and $17^{a-3} \mid \pi+1$.

Proof. If $p$ is a prime and $p \leqslant 113$, then $p \not \pm 1$ (17) except for $p=103$. Hence by Lemmas 1,2 and 4 if $17 \mid \sigma\left(p_{i}^{a_{i}}\right)$ for $1 \leqslant i \leqslant 7$, then $i=7, p_{7}=103$ and $17 \nmid \sigma\left(p_{8}^{a_{8}}\right)$.

Suppose $p_{7} \neq 103$. Then $17^{a} \mid \sigma\left(p_{8}^{a_{8}} p_{9}^{a_{9}} p_{10}^{a_{10}}\right)$. Since $a \geqslant 8,17^{3} \mid \sigma\left(p_{i}^{a_{i}}\right)$ for some $8 \leqslant i \leqslant 10$. If $p_{i} \equiv 1$ (17), then $17^{3} \mid a_{i}+1$, and by Lemma $3 N$ would have at least three more primes $\equiv 1$ (17), a contradiction. Hence $p_{i} \equiv-1$ (17) and $p_{i}=\pi$. Then by the same lemma $17^{2} \nmid \sigma\left(p_{j}^{a_{j}}\right)$ for $j \neq i, 8 \leqslant j \leqslant 10,17^{a-2} \mid \sigma\left(p_{i}^{a_{i}}\right), 17^{2} \nmid a_{i}+1$, 
and $17^{a-3} \mid p_{i}+1$ by Lemma 2 . By Lemma $5 p_{i} \nmid \sigma\left(17^{a}\right)$, and by Corollary $6 \sigma\left(17^{a}\right)$ has at least one prime factor $\geqslant 100129$.

The same arguments hold if $p_{7}=103$ because $17 \nmid \sigma\left(p_{8}^{a_{8}}\right)$. Q.E.D.

LEMMA 8. If $5^{a} \| N$ and $a \geqslant 14$, then $a \geqslant 16, p_{9} \geqslant 100129$ and $p_{10} \geqslant 579281$.

Proof. We showed that $a \neq 14$ in the proof of Corollary 6. Suppose $p^{b} \| N, p \equiv 1$ (5) and $p \leqslant 107$. Then $p=11,31,41,61,71$, or 101 . If $5 \mid \sigma\left(p^{b}\right)$, then by Lemma 2 $\sigma\left(p^{4}\right) \mid \sigma\left(p^{b}\right)$. Since $131 \cdot 21491\left|\sigma\left(61^{4}\right), 211 \cdot 2221\right| \sigma\left(71^{4}\right)$ and $31 \cdot 391 \cdot$ $1381 \mid \sigma\left(101^{4}\right)$, it is easy to show that if $5 \mid \sigma\left(p^{b}\right), p \neq 61,71$, or 101 .

Suppose $5 \mid \sigma\left(41^{b}\right)$. Then $579281 \mid \sigma\left(41^{4}\right)$. Since the order of $5 \bmod 579281$ is 72410 and $a$ is even, $579281 \nmid \sigma\left(5^{a}\right)$, and $\sigma\left(5^{a}\right)$ has at least one prime factor $\geqslant 100129$ by Corollary 6 . Hence we may assume that $5 \nmid \sigma\left(41^{b}\right)$.

Since $3001 \cdot 3221 \cdot 24151 \mid \sigma\left(11^{24}\right)$ and $101 \cdot 4951 \cdot 17351 \mid \sigma\left(31^{24}\right), 5^{2} \nmid \sigma\left(11^{b}\right)$ and $5^{2} \nmid \sigma\left(31^{b}\right)$.

Suppose $5 \mid \sigma\left(11^{b}\right)$. Then $3221 \mid \sigma\left(11^{4}\right)$, and if $3221^{c} \| N, 5^{2} \nmid \sigma\left(3221^{c}\right)$ because $151 \cdot 601 \cdot 1301 \cdot 1601 \mid \sigma\left(3221^{24}\right)$. Similarly, if $5 \mid \sigma\left(31^{b}\right)$, then $17351 \mid \sigma\left(31^{4}\right)$, and if $17351^{c} \| N, 5^{2} \nmid \sigma\left(17351^{c}\right)$ because $101 \cdot 2351 \mid \sigma\left(17351^{24}\right)$.

Suppose $p^{b} \| N, p \equiv-1(5)$, and $p \leqslant 107$. Then $p=19,29,59,79$, or 89 , and by Lemma $1 p \neq \pi$. Hence by Lemma $25 \nmid \sigma\left(p^{b}\right)$.

In summary if $p^{b} \| N, p \leqslant 107$, and if $5 \mid \sigma\left(p^{b}\right)$, then $p=11$ or 31 , in which case $q^{c} \| N$ where $q=3221$ or 17351 and $5^{2} \nmid \sigma\left(q^{c}\right)$.

Now we will show that $5^{a-8} \mid \pi+1$. Suppose three $p_{i} \equiv 1(5)$ for $1 \leqslant i \leqslant 7$. Then $p_{3}=11, p_{6}=31$ and $p_{7}=41$, and it is easy to show that $41<p_{8} \leqslant 61$. Hence $5 \nmid \sigma\left(p_{8}^{a_{8}}\right)$. Since $p_{10} \geqslant 100129$, the above summary shows that $5^{2} \nmid \sigma\left(\prod_{i=1}^{8} p_{i}^{a_{i}}\right)$. Suppose $5^{a} \| \sigma\left(p_{9}^{a_{9}} p_{10^{0}}^{a_{0}}\right)$. By a similar argument used in the proof of Lemma 7 we have for $i=9$ or $105^{a-4} \mid \sigma\left(p_{i}^{a_{i}}\right), p_{i}=\pi$, and $5^{a-6} \mid \pi+1$. Suppose $5^{a-1} \| \sigma\left(p_{9}^{a_{9}} p_{10}^{a_{10}}\right)$. Then $p_{9}=3221$ or 17351 and $5^{2} \nmid \sigma\left(p_{9}^{a_{9}}\right), 5^{a-2} \mid \sigma\left(p_{10}^{a_{10}}\right), p_{10}=\pi$, and $5^{a-4} \mid \pi+1$.

Similar arguments show that if two $p_{i} \equiv 1(5)$ for $1 \leqslant i \leqslant 7$, then $5^{a-8} \mid \pi+1$ and that if $p_{i} \equiv 1(5)$ for $1 \leqslant i \leqslant 7$, then $5^{a-5} \mid \pi+1$.

Since $a \geqslant 16$ and $5^{a-8} \mid \pi+1, \pi \nmid \sigma\left(5^{a}\right)$ by Lemma $5, \pi \geqslant 2 \cdot 5^{a-8}-1>7 \cdot 10^{5}$ $>579281$, and Lemma 8 follows from Corollary 6. Q.E.D.

Corollary 8. Suppose $5^{a} \| N, a \geqslant 14$, and $579281 \nmid N$ if $41 \mid N$. Then $a \geqslant 16$, $p_{9} \geqslant 100129, p_{10} \geqslant 2 \cdot 5^{a-8}-1>7 \cdot 10^{5}$, and $5^{a-8} \mid \pi+1$.

The next lemma is due to McDaniel [6].

LEMMA 9. Suppose $a \geqslant 2, a+1$ is a prime, and $p$ is a prime.

(a) If $p^{2} \mid \sigma\left(5^{a}\right)$, then $p>2^{29}$.

(b) If $p^{2} \mid \sigma\left(17^{a}\right)$, then $p>2^{27}$ or $p=48947$.

LEMMA 10. Suppose $p^{a} \| N, q\left|\sigma\left(p^{b}\right), b+1\right| a+1, q \leqslant 107$, and $q, b+1$ are primes. Then

(a) If $p=5, q=11,31,59$, or 71 .

(b) If $p=17, q=47,59$, or 83 .

Proof. Suppose $p=5$ or 17, and $d$ is the order of $p \bmod q$. Then $p^{d} \equiv 1(q)$, and $d \mid b+1$. Since $p \neq 1(q), d>1$, and $d=b+1$ because $b+1$ is an odd prime. The order $d$ is not an odd prime except for those $q$ stated above. Q.E.D. 
Lemma 11. Suppose $17^{a} \| N, a \geqslant 8$, and $307 \nmid N$. If $p_{8}<1000$, then $a \geqslant 10$, $p_{9} \geqslant 25646167$, and $p_{10}>8 \cdot 10^{8}$.

Proof. Since $307 \mid \sigma\left(17^{8}\right), a \geqslant 10$, and by Lemma $7,17^{a-3} \mid \pi+1$ and $p_{10} \geqslant$ $2 \cdot 17^{a-3}-1>8 \cdot 10^{8}$. Suppose $p_{8}<1000$ and $100129 \leqslant p_{9}<25646167<2^{27}$. Choose $b$ such that $b+1$ is a prime and $b+1 \mid a+1$. Then $\sigma\left(17^{b}\right) \mid \sigma\left(17^{a}\right)$, and $b \neq 2$, 4 , or 6 because $307\left|\sigma\left(17^{2}\right), 88741\right| \sigma\left(17^{4}\right)$ and $25646167 \mid \sigma\left(17^{6}\right)$. Hence $b \geqslant 10$. If $1 \leqslant i \leqslant 7$ and $p_{i} \mid \sigma\left(17^{b}\right)$, then by Lemmas $4,10 i=7$ and $p_{7}=47,59$, or 83. Since $\pi=p_{10} \nmid \sigma\left(17^{a}\right)$ by Lemma 5 , we have $\sigma\left(17^{b}\right) \mid p_{7} p_{8} p_{9}$ by Lemma 9 . Then $\sigma\left(17^{10}\right) \leqslant 83 \cdot 1000 \cdot p_{9}$, or $p_{9}>25646167$, a contradiction. Hence $p_{9} \geqslant 25646167$. Q.E.D.

COROLlaRY 11. If $p_{7} \leqslant 29$ and $p_{8}<6203$, then $a \geqslant 10, p_{9} \geqslant 25646167$, and $p_{10}>8 \cdot 10^{8}$.

Proof. As in Lemma $11 \sigma\left(17^{b}\right) \mid p_{8} p_{9}$. Then $\sigma\left(17^{10}\right) \leqslant 6203 \cdot p_{9}$, or $p_{9}>25646167$, a contradiction. Q.E.D.

Lemma 12. Suppose $5^{a} \| N, a \geqslant 14,579281 \nmid N$ if $41 \mid N$, and $p \nmid N$ if $p=31,71$, 191,409 , or 19531. If $p_{8} \leqslant 41$, then $a \geqslant 22, p_{9} \geqslant 12207031$, and $p_{10} \geqslant 2 \cdot 5^{a-8}-1>$ $10^{10}$.

Proof. $a \geqslant 22$ because $a \neq 14$ as before, $409\left|\sigma\left(5^{16}\right), 191\right| \sigma\left(5^{18}\right)$, and $19531 \mid \sigma\left(5^{20}\right)$. The rest of the proof is similar to that of Lemma 11 using $\sigma\left(5^{10}\right)=$ 12207031 and $\sigma\left(5^{12}\right)=307175781$. Q.E.D.

Corollary 12. If $p_{7} \leqslant 29$ and $p_{8}<6203$, then $a \geqslant 22, p_{9} \geqslant 12207031$, and $p_{10} \geqslant 2 \cdot 5^{a-8}-1>10^{10}$.

Lemma 13. Suppose $5^{a} \| N, a=10$ or $12, p_{8} \leqslant 151, p_{9}>3011$, at most two $p_{i} \equiv 1$ (5) for $1 \leqslant i \leqslant 8, p_{i}=11,31,41$, or 151 if $p_{i} \equiv 1$ (5) and $1 \leqslant i \leqslant 8, p_{i}=19,29,59$, $79,89,109$, or 149 if $p_{i} \equiv-1(5)$ and $1 \leqslant i \leqslant 8, p \nmid N$ if $p=131,3221$, or 17351, and $579281 \nmid N$ if $41 \mid N$. Then $p_{9} \geqslant 3 \cdot 10^{6}$, and $p_{10} \geqslant 12207031$.

Proof. Suppose $3011<p_{9}<3 \cdot 10^{6}$. Then $p_{10}=\sigma\left(5^{a}\right)=12207031$ or 305175781 , and $5 \nmid \sigma\left(p_{10^{0}}^{a_{10}}\right)$ because $131 \mid \sigma\left(12207031^{4}\right)$ and $3011 \mid \sigma\left(305175781^{4}\right)$. Suppose $5 \mid \sigma\left(p_{i}^{a_{i}}\right), \quad 1 \leqslant i \leqslant 8$. Since $3221\left|\sigma\left(11^{4}\right), 17351\right| \sigma\left(31^{4}\right), 579281 \mid \sigma\left(41^{4}\right)$ and $104670301 \mid \sigma\left(151^{4}\right), p_{\mathrm{i}} \neq 1$ (5). Since $p_{i} \neq \pi$ if $p_{i}=19,29,59,79,89$, and 149 by Lemma 1 , we have $p_{8}=109=\pi$ by Lemmas 2 , 4. If $5^{4} \mid \sigma\left(p_{8}^{a_{8}}\right)$, then $5^{3} \mid a_{8}+1$ by Lemma 2 , and $N$ would have at least six prime factors $\equiv 1$ (5), a contradiction. Hence $5^{4} \nmid \sigma\left(p_{8}^{a_{8}}\right)$, and so $5^{a-3} \mid \sigma\left(p_{9}^{a_{9}}\right), p_{9} \neq \pi, p_{9} \equiv 1$ (5) and $N$ would have at least 7 more prime factors $\equiv 1(5)$, a contradiction. Hence $5 \nmid \sigma\left(p_{i}^{a_{i}}\right)$ for $1 \leqslant i \leqslant 8$.

Then $5^{\mathrm{a}}\left|\sigma\left(p_{9}^{a_{9}}\right), p_{9}=\pi, 5^{2} \nmid a_{9}+1,5^{a-1}\right| p_{9}+1$, and $p_{9} \geqslant 2 \cdot 5^{a-1}-1>$ $3 \cdot 10^{6}$, a contradiction. Q.E.D.

Definition. Suppose $M=\prod_{i=1}^{r} p_{i}^{a_{i}}$. Then

$$
\begin{gathered}
S(M)=\sigma(M) / M, \\
a\left(p_{i}\right)=\min \left\{a_{i} \mid p_{i}^{a^{i}+1}>10^{10} \text { where } p_{i}^{a}\right. \text { satisfies the restrictions }
\end{gathered}
$$

$$
\text { implied by Lemma } 1\} \text {, }
$$




$$
b_{i}= \begin{cases}a_{i} & \text { if } a_{i}<a\left(p_{i}\right) \\ a\left(p_{i}\right) & \text { if } a_{i} \geqslant a\left(p_{i}\right)\end{cases}
$$

Lemma 14. Suppose $M=\prod_{i=1}^{10} p_{i}^{b_{i}}$. Then $0 \leqslant \log 2-\log S(M)<10 \cdot 10^{-10}$.

Proof. Suppose $p^{a} \| N$ and $a \geqslant a(p)$. Then

$$
\begin{aligned}
0 & \leqslant \log S\left(p^{a}\right)-\log S\left(p^{a(p)}\right)<\log \frac{p}{p-1}-\log \frac{p^{a(p)+1}-1}{p^{a(p)}(p-1)} \\
& =\log \frac{p^{a(p)+1}}{p^{a(p)+1}-1}=\log \left(1+\frac{1}{p^{a(p)+1}-1}\right) \\
& <\frac{1}{p^{a(p)+1}-1} \leqslant 10^{-10} .
\end{aligned}
$$

Hence

$$
\begin{aligned}
0 & \leqslant \log S(N)-\log S(M) \\
& \leqslant \sum_{i=1}^{10}\left|\log S\left(p_{i}^{a_{i}}\right)-\log S\left(p_{i}^{b_{i}}\right)\right|<10 \cdot 10^{-10}
\end{aligned}
$$

Q.E.D.

The proof of the next two lemmas is easy.

LEMMA 15. If $q$ is a prime, $q \mid \sigma\left(p_{i}^{a_{i}}\right)$ for some $1 \leqslant i \leqslant 7$ with $a_{i}<a\left(p_{i}\right)$, and if $q \leqslant p_{7}$, then $q=2$ or $q=p_{i}$ for some $1 \leqslant i \leqslant 7$.

Lemma 16. Suppose $M=\prod_{i=1}^{7} p_{i}^{b_{i}}$ and $L=M \cdot \prod_{j=1}^{r} q_{j}^{c_{j}}$ where $q_{j}$ is a prime, $q_{j}>p_{7}, q_{j} \mid \sigma\left(p_{i}^{b_{i}}\right)$ for some $1 \leqslant i \leqslant 7$ with $b_{i}<a\left(p_{i}\right), q_{1}<\cdots<q_{r}$, and $c_{j}$ is the minimum allowable power of $q_{j}$ determined by Lemma 1 . If there is no such $q_{j}$, then $r=0$ and the product is defined to be 1 . Then

(a) $r \leqslant 3$ and $\log S(L) \leqslant \log 2$.

(b) If $r=3$, then $p_{8}=q_{1}, p_{9}=q_{2}, p_{10}=q_{3}$ and

$$
\log 2<\log S(M)+7 \cdot 10^{-10}+\sum_{j=1}^{3} \log q_{j} /\left(q_{j}-1\right) .
$$

(c) If $r=2$ and $q_{2}<100129$, then $p_{8}=q_{1}, p_{9}=q_{2}$ and

$$
\log 2<\log S(M)+7 \cdot 10^{-10}+\sum_{j=1}^{2} \log q_{j} /\left(q_{j}-1\right)+\log 100129 / 100128 \text {. }
$$

LEMMA 17. $p_{8}<3011$.

Proof. Suppose $p_{8} \geqslant 3011$. We used a computer (PDP 11/70 at the University of Toledo) to find $M=\prod_{i=1}^{7} p_{i}^{b_{i}}$ satisfying Lemmas $1,4,15,16, \log S(M)<\log 2$, and

$$
\begin{aligned}
\log 2<\log S(M)+7 \cdot 10^{-10} & +\log 3011 / 3010 \\
& +\log 3019 / 3018+\log 100129 / 100128 .
\end{aligned}
$$


The results were:

$$
\begin{aligned}
& 5^{14} 7^{12} 11^{10} 13^{9} 17^{8} 23^{a_{6}} 29^{6}, \\
& 5^{14} 7^{12} 11^{10} 13^{9} 17^{6} 23^{a_{6}} 29^{6}, \\
& 5^{14} 7^{12} 11^{10} 13^{6} 17^{8} 23^{a_{6}} 29^{6}, \\
& 5^{12} 7^{12} 11^{10} 13^{9} 17^{8} 23^{a_{6}} 29^{6}, \\
& 5^{10} 7^{12} 11^{10} 13^{9} 17^{8} 23^{a_{6}} 29^{6},
\end{aligned}
$$

where $a_{6}=6$ or 8 . Since

$$
\frac{5}{4} \frac{7}{6} \frac{11}{10} \frac{13}{12} \frac{17}{16} \frac{23}{22} \frac{29}{28} \frac{6203}{6202} \frac{6211}{6210} \frac{100129}{100128}<2,
$$

$3011 \leqslant p_{8}<6203$. By Corollaries 11 and $12 p_{9} \geqslant \min \{25646167,12207031\}$ and $p_{10} \geqslant \min \left\{8 \cdot 10^{8}, 10^{10}\right\}$. Then $N$ is not OP because

$$
\frac{5}{4} \frac{7}{6} \frac{11}{10} \frac{13}{12} \frac{17}{16} \frac{23}{22} \frac{29}{28} \frac{3011}{3010} \frac{12207031}{12207030} \frac{800000000}{799999999}<2 \text {. Q.E.D. }
$$

The proof of the next lemma is also easy.

LEMMA 18. Suppose $M=\prod_{i=1}^{9} p_{i}^{b_{i}}, q=\max \{p \mid p$ is a prime and $\log S(M)+$ $\log S\left(p^{a}\right) \geqslant \log 2$ where $a$ is the minimum allowable power of $\left.p\right\}$ and $r=\min \{p \mid p$ is $a$ prime and $\left.\log S(M)+9 \cdot 10^{-9}+\log p /(p-1)<\log 2\right\}$. Then $q<p_{10}<r$; in particular, if there are no primes between $q$ and $r, N$ is not $O P$.

LEMMA 19. $p_{9}<3011$.

Proof. Suppose $p_{8}<3011 \leqslant p_{9}$. We used a computer to find $M=\prod_{i=1}^{8} p_{i}^{b_{i}}$ satisfying Lemmas $1,4,7,8,15,16, \log S(M)<\log 2$, and

$$
\log 2<\log S(M)+8 \cdot 10^{-10}+\log 3011 / 3010+\log 100129 / 100128 .
$$

There were seventy-two such $M$ 's. However, none of them satisfied Lemmas 11,12 and 13 except

$$
5^{2} 7^{12} 11^{10} 13^{10} 19^{10} 23^{8} 31^{6} 59^{6} \text {. }
$$

It is easy to show that $7753 \leqslant p_{9} \leqslant 8389, a_{2} \geqslant 22\left(\sigma\left(7^{12}\right)\right.$ is a prime), $a_{3} \geqslant 16$, $a_{4} \geqslant 16, a_{5}=10$ or $\geqslant 16$ (if $\sigma\left(19^{10}\right)$ is a prime, $a_{5} \geqslant 16$ ), $a_{6} \geqslant 12, a_{7} \geqslant 12$, and $a_{8} \geqslant 12$. Then for each $p_{9}$ with $7753 \leqslant p_{9} \leqslant 8389$ Lemma 18 is not satisfied. Hence $N$ is not OP. Q.E.D.

Proof of Theorem. By Lemma $19 p_{9}<3011$. We used a computer to find $M=\prod_{i=1}^{9} p_{i}^{b_{i}}$ satisfying Lemmas $1,4,7,8,15,16, \log S(M)<\log 2$, and

$$
\log 2<\log S(M)+9 \cdot 10^{-10}+\log 100129 / 100129 .
$$

There were thirty-nine such $M$ 's; however, none of them satisfied Lemma 18 . Hence $N$ is not OP. Q.E.D.

Acknowledgement. The author would like to thank Professor P. Hagis, Jr. for supplying the table in Lemma 6.

Department of Mathematics

The University of Toledo

Toledo, Ohio 43606 
1. J. E. Z. Chein, An Odd Perfect Number Has at Least 8 Prime Factors, Ph.D. Thesis, Pennsylvania State University, 1979.

2. P. Hagis, JR., "Outline of a proof that every odd perfect number has at least eight prime factors," Math. Comp., v. 35, 1980, pp. 1027-1032.

3. P. HAGis, JR. \& W. L. MCDANIEL, "On the largest prime divisor of an odd perfect number. II," Math. Comp., v. 29, 1975, pp. 922-924.

4. M. Kishore, "Odd perfect numbers not divisible by 3 are divisible by at least ten distinct primes," Math. Comp., v. 31, 1977, pp. 274 - 279.

5. M. KiSHORE, "Odd integers $N$ with five distinct prime factors for which $2-10^{-12}<\sigma(N) / N<2$ $+10^{-12, " ~ M a t h . ~ C o m p ., ~ v . ~ 32, ~ 1978, ~ p p . ~ 303-309 . ~}$

6. W. L. MCDANIEL, “On multiple prime divisors of cyclotomic polynomials," Math. Comp., v. 28, 1974, pp. 847-850.

7. C. Pomerance, “Odd perfect numbers are divisible by at least seven distinct primes," Acta Arith., v. $25,1973 / 74$, pp. 265-300.

8. C. Pomerance, “The second largest prime factor of an odd perfect number," Math. Comp., v. 29, 1975, pp. 914-921. 